\title{
Calculation of Roadlighting Photometric Parameters Under Mesopic Conditions
}

\begin{abstract}
At the present the mesopic photometry is hot topic in lighting engineering especially in the public lighting. The mesopic photometry is used in particular to consider roadlighting photometric parameters where mesopic vison is relevant to car drivers and pedestrians. Nowadays, works in the framework of the joint technical committee JTC001 at CIE is focused on mesopic photometry application. Within JTC001 there are two groups which are assigned to solve the stated problems. The first of them concerns fundamental problems on the processes of mesopic vision. e. g. adaptation luminance, field of view, pupil diameter of the eye etc. The second group follows the results of the first group. It should provide practical solutions in the mesopic photometry. One of this topic is devoted to roadlighting calculations. Methodology of roadlighting calculations is laid down in the document CIE 140:2000 which is currently under revision at CIE Division 4 (TC 4-15). Calculations are still based on photopic vision, i. e. photopic photometric quantities what disqualifies some innovative light sources mounted into luminaires which could be more efficient than traditional light sources if taking into account the mesopic conditions. It means that the old approach suppresses the usage of these luminaires because it does not consider appropriately the processes in the mesopic region where efficiency of the human eye is different. It depends on particular situation on the road and it is influenced by many other parameters. Therefore, new approach in the roadlight calculation emerged as a necessity for lighting engineers, who deal with roadlight, to consider mesopic conditions. Some preliminary results have been published in papers dealing with calculations based on the S/P ratio. This paper deals with roadlighting calculations assuming the mesopic photometry applied for various situations on the road with different roadlight luminaires and different light sources. Detailed analyses of possibilities how to use the mesopic photometry in practical calculations of photometric parameters of roadlighting for selected situations and luminaires are also presented in the paper.
\end{abstract}

Keywords: Public lighting, Roadlighting calculation, Mesopic photometry.

\section{Introduction}

The mesopic photometry is historically very well known from the beginning of previous century. Although the physiological processes in the human eye are very-well known the real application of the mesopic photometry into the practice is still not possible due to different reasons. Many years scientists over the world were trying to found connections between vision in the mesopic photometry and photometric quantities. Especially they wanted to establish clear relation between brightness of the object or scene as qualitative aspect of the vision of observer and quantitative expression by means of luminance. These attempts were not every time successful because response of rods and cones in the mesopic region work in different way and they were not allowed establish this relation in clear way. Even more additivity law what is working in good way in photopic and scotopic region was not possible to use for expression of photometric quantities. At the beginning of 90 's of $20^{\text {th }}$ century started slightly appear another approach how to describe mesopic vision with system which should follow additivity law through vision performance. Therefore in 2010 was at the CIE level accepted unified recommended system for mesopic photometry completed by release of CIE document 191:2010 Recommended System for Photometry Based on Visual Performance [1] based on visual performance of observer. This system allow to use additivity law what is the one of main requirement for this system and also allow to express human eye responsivity function $V_{\text {mes }}(\lambda)$ in the mesopic region. This function is not unique over the whole region of mesopic photometry i.e. between luminance levels $0,005 \mathrm{~cd} \cdot \mathrm{m}^{-2}$ and $5 \mathrm{~cd} \cdot \mathrm{m}^{-2}$ because that function is dynamically changing due to different contribution of rods and cones to the vision at different luminance level in respect with adaptation luminance. This combination of rods and cones is described in the present system by linear combination of functions $V(\lambda)$ and $V^{\prime}(\lambda)$ weighted by parameter $m$ which express the measure of contribution either rods or cones to the visual perception and has a value between 0 and 1 . Then it is possible to describe functions in order to determine the relevant values and be applicable to CIE system for mesopic photometry described in the document CIE 191:2010 by relations (1) and (2).

$$
M(m) \cdot V_{\text {mes }}(\lambda)=m \cdot V(\lambda)+(1-m) V^{\prime}(\lambda) \quad \text { for } 0 \leq m \leq 1
$$

and

$$
L_{\mathrm{mes}}=\frac{683}{V\left(\lambda_{0}\right)} \cdot \int L_{\mathrm{e}}(\lambda) \cdot V_{\mathrm{mes}}(\lambda) \mathrm{d} \lambda
$$

where

$$
\begin{array}{ll}
M(m) & \text { is normalising function such that } V_{\text {mes }}(\lambda) \\
& \text { attains a maximum value of } 1 \\
L_{\text {mes }} & \text { is mesopic luminance } \\
V_{\text {mes }}\left(\lambda_{0}\right) & \text { is the value of } V_{\text {mes }}(\lambda) \text { at the } 555 \mathrm{~nm} \\
L_{\mathrm{e}}(\lambda) & \text { is the spectral radiance in W. } \mathrm{m}^{-2} \cdot \mathrm{sr}^{-1} \cdot \mathrm{nm}^{-1}
\end{array}
$$

If $L_{\text {mes }} \leq 0,005 \mathrm{~cd} \cdot \mathrm{m}^{-2}$ then $\mathrm{m}=0$ and if $L_{\text {mes }} \geq 5 \mathrm{~cd} \cdot \mathrm{m}^{-2}$ then $\mathrm{m}=1$. The coefficient $\mathrm{m}$ and the mesopic luminance $L_{\text {mes }}$ can be calculated using iterative approach as follows

$$
\begin{aligned}
& m_{0}=0,5 \\
& L_{m e s, n}=\frac{m_{(n-1)} L_{p}+\left(1-m_{(n-1)}\right) L_{s} V^{\prime}\left(\lambda_{0}\right)}{m_{(n-1)}+\left(1-m_{(n-1)}\right) V^{\prime}\left(\lambda_{0}\right)} \\
& m_{n}=a+b \log _{10}\left(L_{m e s, n}\right) \quad \text { for } \quad 0 \leq m \leq 1
\end{aligned}
$$

where

$L_{p} \quad$ is the photopic luminance

$L_{s} \quad$ is the scotopic luminance

$V^{\prime}(\lambda)$ is the value of scotopic spectral luminous efficiency at $\lambda_{0}=555 \mathrm{~nm}$

$a, b$ are parameters with values $a=0,7670$ and $b=$ 0,3334 
Into both of relationships (1) and (2) are needed to know values of luminances which can be determined for example from photometric measurement or assuming these parameters from photometric data of luminaire used in the calculation.

\section{Adaptation luminance}

At the present the most of research work in the mesopic photometry field is focused on establishment appropriate methods how to determine adaptation luminance. Assuming drivers at outdoor lighting conditions the light scene in the field of view of the observer is diverse due to different objects with various luminance levels, moving objects etc. All these things influence also adaptation state of visual system of the observer. It should be noted that only determination of adaptation luminance prevents implementation of the recommended system for mesopic photometry into the practice. Therefore nowadays they are addressed in the various research projects as problems regarding the establishment of areas of adaptation of visual system in the field of view of the observer under the conditions of mesopic vision, to be taken into account for determining an adaptation luminance with respect to specific situations visual tasks of the observer under defined lighting conditions. Generally the determination of the adaptation luminance depends on the proper use of a particular spectral responsivity of the human eye to be considered. Then subsequent lighting calculations can use results of determination of adaptation luminance as well as they can serve as for appropriate measurement system of the mesopic photometric quantities. Taking account of these effects in different light conditions in the observer's field is still looking for a suitable system for clear identification of adaptation brightness. Described recommended system for mesopic photometry is based on model visual performance of the observer i.e. means taking into consideration the impact of the central and peripheral vision [2,3]. These visual tasks are most essential for lighting conditions of outdoor lighting systems at night driving a car. The car driver is subject to different visual tasks, which in the process of vision lighting conditions, external lighting systems occur. Under these conditions have to be considered processes of the visual system which directly affecting visual perception. They are based on the consideration of luminance contrast of the observed objects to the brightness of the background, which exactly corresponds to the vision-performance and not to the contrast of brightness of objects in the visual field of the observer. The basic visual tasks are based on three fundamental issues as follows

a) Can the object in the visual field observer clearly recognized?

b) How fast can the observer recognize the subject?

c) What is the object in the visual field of the observer?

All of these questions should be assumed at determination of adaptation luminance. Recently new approach about of calculation of adaptation luminance based on analysis of luminance distribution of light scene measured by image photometer was developed in Japan [4]. It takes into account all three assumptions as answers on the questions above i.e. direction of line of sight of observer, area for which will be computed photometric parameters, surrounding luminance effect, luminance distribution of the scene and area under consideration to be illuminated. These aspects are described by means of the functions LD (luminance distribution), EM (eye movement), SLE (surrounding luminance effect) and AOM (area of measurement). All of these functions are assumed to be independent. Each of this function is based on coordinate system of eye retina of observer and object plane described by two angles $\varphi, \vartheta$. This coordinate system is depicted in the picture Fig.1.

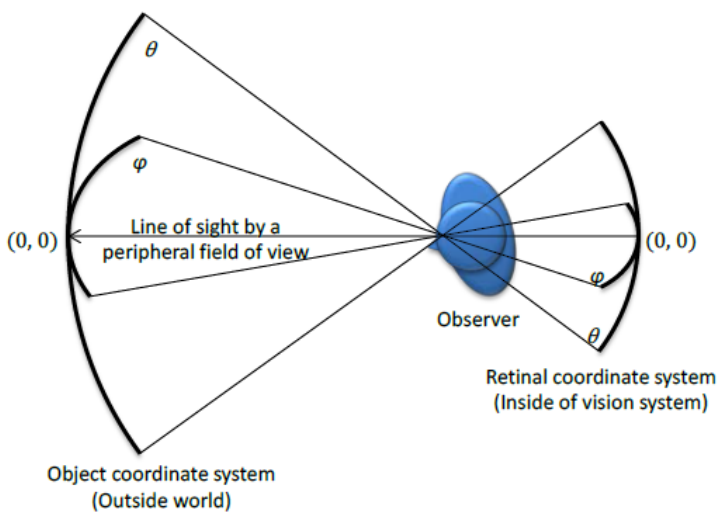

Fig.1. Object and retinal coordinate system of the observer [4]

The simulation method to calculate the adaptation luminance consists of the following four steps.

1. Effective luminance distribution calculation

2. Adaptation luminance distribution calculation

3. AOM hit probability distribution calculation

4. Adaptation luminance calculation

By means of convolutions of described functions LD, EM, SLE and AOM in combination with integrations (see reference [4] for more details) the adaptation luminance of $A O M$, which is the average adaptation luminance weighted with the AOM hit probability distribution, is derived as

$$
L_{a, A O M}=\frac{\iint L_{a}(\theta, \varphi) \cdot P_{A O M}(\theta, \varphi) \mathrm{d} \varphi \mathrm{d} \theta}{\iint P_{A O M}(\theta, \varphi) \mathrm{d} \varphi \mathrm{d} \theta}
$$

In Japan were investigated 16 light scenes of walksides and park areas [4]. For each area was calculated adaptation luminance from luminance distribution obtained by image photometer based on CCD element. From the luminance distribution of the scene was calculated adaptation luminance for each area of interest which was illuminated by different luminaires.

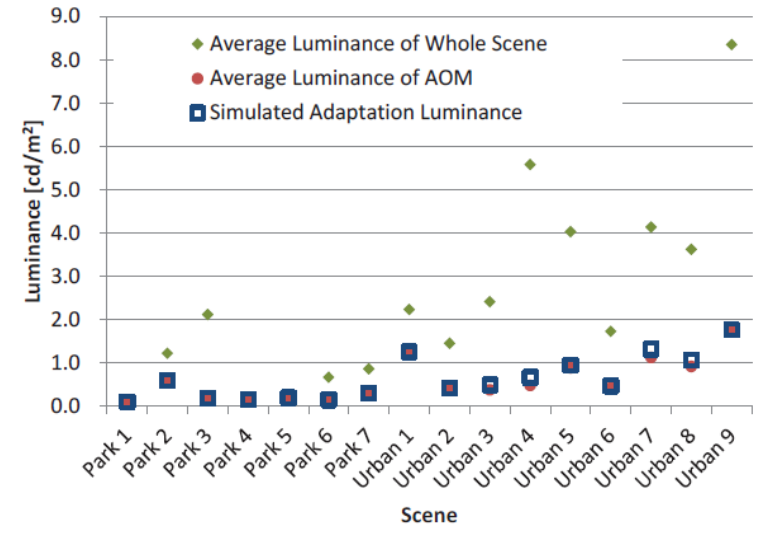

Fig.2. Simulated adaptation luminance / small EM [4] 
After the calculation based on the proposed model was found good predictor which can be used for determination of adaptation luminance directly from luminance distribution. Investigation was made for different EM functions which describes the measure of eye movement by means of two dimensional Gaussian functions. Consideration was done for small, medium and large change of the line of sight of the observer (Fig.2, Fig.3 and Fig.4).

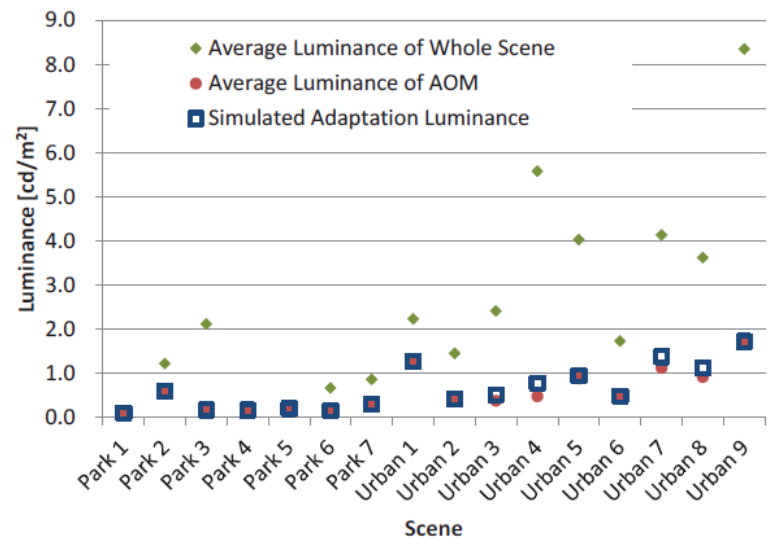

Fig.3. Simulated adaptation luminance / medium EM [4]

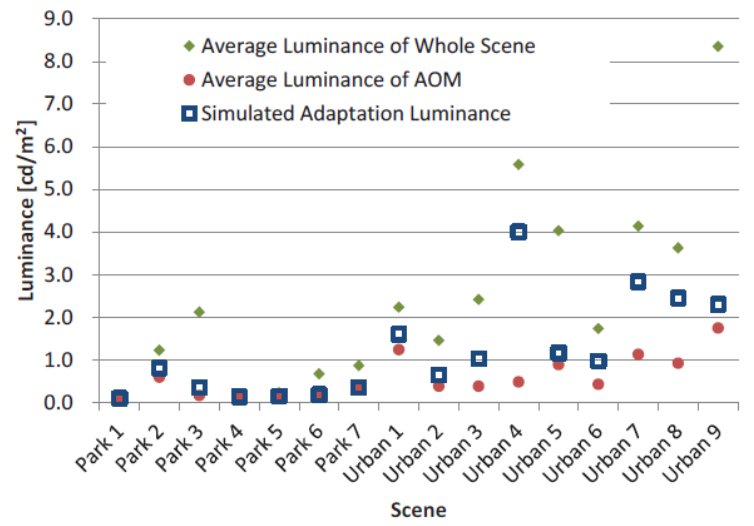

Fig.4. Simulated adaptation luminance / large EM [4]

In the graphs of the simulation results are depicted also predictors which can be used as directly for determination of adaptive luminance without following of complex mathematic process. As good predictor of adaptation luminance seems avaregae luminance of area of interest to be illuminated (AOM) for small and medium EM, but for large EM it was shown that for some areas it does not match so well with calculation of adaptation luminance. Therefore it should be investigated in more detail for other scenes and various EM functions.

\section{Roadlight calculation}

In the roadlighting calculation depending on the desired application is used as a design criterion for a good lighting system photometric quantities luminance and lluminance. The luminance is used to evaluate and lighting design carriage way where it is possible to define precisely the reflectance of the surface of the road and the position of the observer. It is also used to draft architectural lighting of buildings and major monuments. The average luminance on the road $\left(L_{0}\right)$ has a major impact on visual comfort, visual performance drivers and barriers affecting the contrast. Impact on visual performance and comfort also has a total uniformity $(\boldsymbol{U})$ ) which is calculated as the ratio of minimum and average luminance value on carriageway. Carrigeways with good overall uniformity may adversely affect the visual comfort of the driver due to poor longitudinal uniformity $\boldsymbol{U}$, which is calculated as the ratio of minimum and maximum luminance in the axis of the lane. For the evaluation of discomfort glare is used the threshold increment $(T I)$ which is characterised by increased resolution of the threshold luminance. The discomfort glare decreases with increasing the average luminance of the background.

The average value of luminance, overall uniformity, and increase the threshold must be calculated for the entire road surface and each position of the observer. The longitudinal uniformity $\boldsymbol{U} \mathbf{I}$ must be calculated for each lane, and the calculation is performed in the axis of the lane. The final value of the average luminance, overall uniformity and longitudinal uniformity must be the lowest among individual cases. On the contrary, increase the threshold value must be the highest value. The intensity of light as a design criterion Light used for pedestrian zones, parks and mixed traffic. Is defined on the comparative plane and distribution of light intensity is evaluated by uniformity defined as a ratio of the minimum and average illuminance value.

The calculation of the brightness of the road surface is considered from a distance of $60 \mathrm{~m}$ in front of the computational area in the middle of the lane of carriageway (Fig.5).

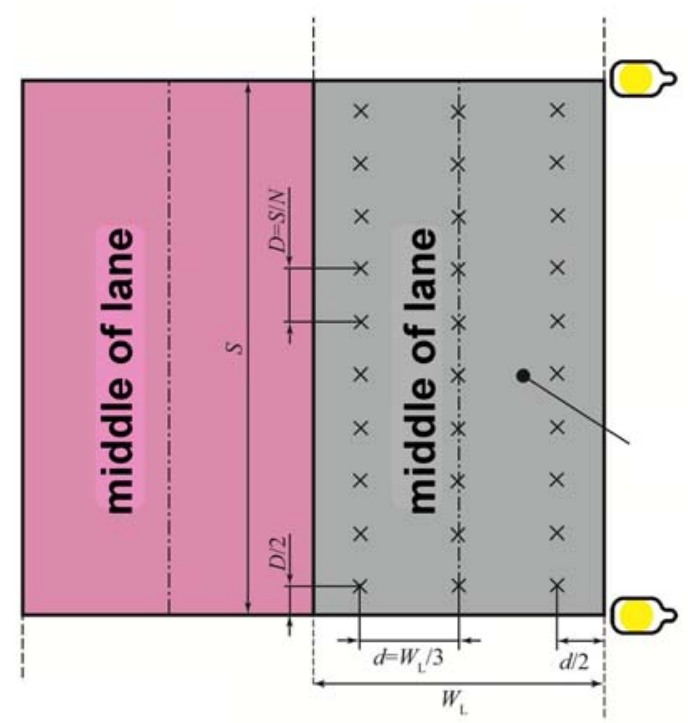

Fig.5. Calculation grid of the carriageway

In the picture Fig. 5 are depicted are geometric parameters which means as follows

$S$ - the distance of the first and last luminaire in the calculation field

WL - width of one lane

$\mathrm{N}$ - number of points in the longitudinal direction

for the $S \leq 30 \mathrm{~m}, \mathrm{~N}=10$,

for $S>30 \mathrm{~m}, \mathrm{~N}=$ the lowest value of the whole to which applies

$\mathrm{D} \leq 3 \mathrm{~m}$

$D$ - distance between two measuring points

$\mathrm{d}$ - distance measurement points in the transverse direction 
Examples of calculation under mesopic condition

In this section are shown some examples of calculation of photometric parameters for particular roadlight situation performed in DIALUX where photopic parameters are computed. From the field measurement by image photometer the luminance distribution was obtained and analysed for adaptation luminance according to estimated average luminance of area of the measurement between two luminaires.

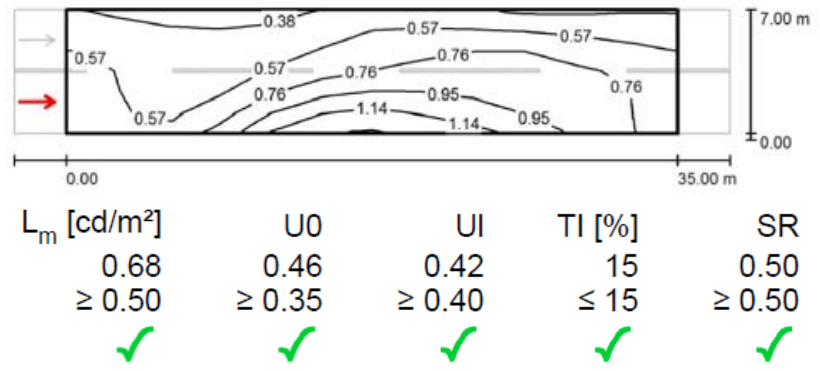

Fig.6. ME5 road class of the carriageway - results DIALUX
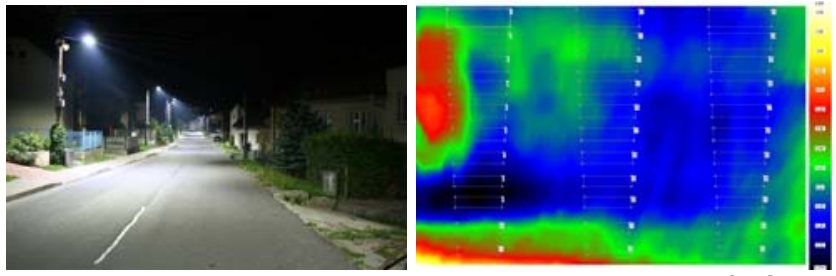

Fig.7. ME5 road class - photo and luminance distribution of AOM

In the pictures Fig.6 and Fig.7 is shown example for calculation of roadclass ME5 computation in the DIALUX software where user can compute only photometric parameters for photopic vision condition. The subject of the measurement was reconstructed roadlighting where LED luminaires $50 \mathrm{~W}$ were installed in height $7,7 \mathrm{~m}$ above the road surface. The luminous intensity distribution curve (LIDC) of the luminaire is depicted in the picture Fig.8.

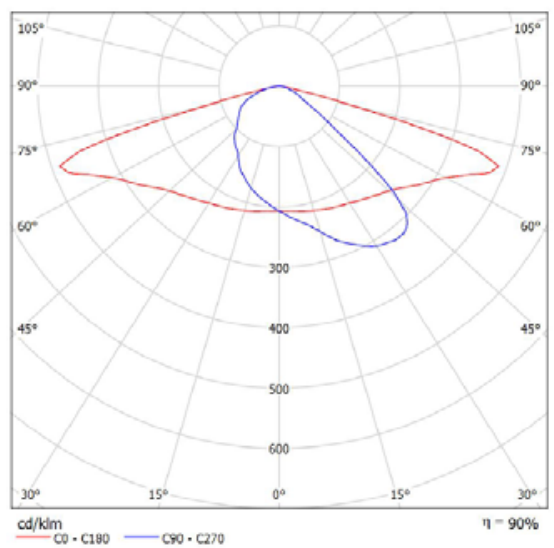

Fig.8. - LIDC of used luminaires in the roadlight installation

From the measured luminance distribution it was computed average luminance of area of the measurement (AOM) and it was taken as adaptation luminance value. Then computation of mesopic luminance was performed for the value of adaptation luminance. As result for roadclass ME5 from the measurement was computed assuming all $L_{\text {mes }}=0,74 \mathrm{~cd} \cdot \mathrm{m}^{-2}$. The measured value was $0,63 \mathrm{~cd} \cdot \mathrm{m}^{-2}$

Same approach as above was used for the other example what was roadclass ME6 where LED luminaires with same luminous intensity distribution curve was used but with different power input 25W.

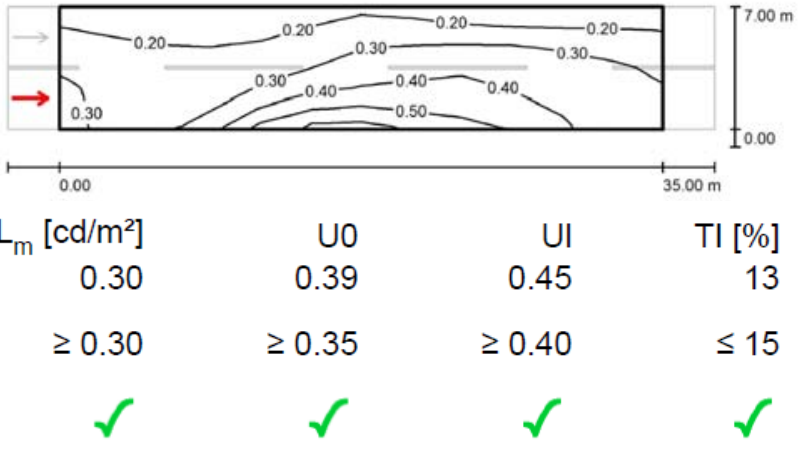

Fig.9. ME6 road class of the carriageway - results DIALUX

As result for roadclass ME5 from the measurement was computed assuming all $L_{\text {mes }}=0,34 \mathrm{~cd} \cdot \mathrm{m}^{-2}$. The measured value was $0,30 \mathrm{~cd} \cdot \mathrm{m}^{-2}$.

\section{Conclusions}

In the paper was presented roadlight calculation under mesopic conditions for particular roadclasses based on current methodology of determination of adaptation luminance which is very important parameter for calculation to be performed. In the examples it can be seen that level of mesopic luminance level of the mounted luminaires in the particular roadclass are higher than photopic luminance level taken from computation tool. It is because of that fact LED luminaires have S/P ratio above 1 i.e. according to mesopic photometry recommended model it should be higher level of luminance at mesopic level. In the future should be investigated more roadclasses with different luminaires and layout of lighting system. In the future work is planned to create classification system for lighting designers with respect to adaptation luminance and luminance distributions to avoid complex computation in the practice to make roadlight calculation easier.

\section{Acknowledgments}

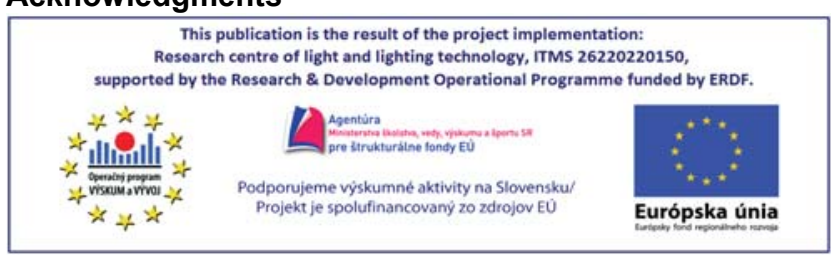

REFERENCES

[1] CIE 2010. CIE 191:2010 Recommended System for Mesopic Photometry Based on Visual Performance. Vienna: CIE

[2] T. Uchida and Y. Ohno: Defining the visual adaptation field for mesopic photometry: Does surrounding luminance affect peripheral adaptation?, Lighting Research and Technology, 2013

[3] Uchida T, Ohno $Y$. Defining the visual adaptation field for mesopic photometry: Does surrounding luminance affect peripheral adaptation?, Lighting Research and Technology 2014; 46: 520-533.

[4] T. Uchida: ADAPTATION LUMINANCE SIMULATION FOR CIE MESOPIC PHOTOMETRY SYSTEM IMPLEMENTATION, Proceedings of 28th CIE Session 2015, Manchester, Great Britain, 29th June - 3rd July 2015, pp.307 - 316

[5] Technical report CIE 140:2000, Road lighting calculation

Authors: Mgr. Roman Dubnička, Slovenská technická univerzita, Fakulta elektrotechniky a informatiky, Ilkovičova 3, Slovak republic, e-mail: roman.dubnicka@stuba.sk 